Revue internationale P.M.E.

Économie et gestion de la petite et moyenne entreprise

\title{
A propos de l'ouvrage d'Henri Mahé de Boislandelle : «Gestion des ressources humaines dans les P.M.E.»
}

\section{Bruno Fabi et Denis Garand}

Volume 2, numéro 1, 1989

URI : https://id.erudit.org/iderudit/1007909ar

DOI : https://doi.org/10.7202/1007909ar

Aller au sommaire du numéro

Éditeur(s)

Presses de l’Université du Québec

ISSN

0776-5436 (imprimé)

1918-9699 (numérique)

Découvrir la revue

Citer cette note

Fabi, B. \& Garand, D. (1989). A propos de l'ouvrage d'Henri Mahé de Boislandelle : «Gestion des ressources humaines dans les P.M.E.». Revue internationale P.M.E., 2(1), 99-108. https://doi.org/10.7202/1007909ar d'utilisation que vous pouvez consulter en ligne. 


\title{
A propos de l'ouvrage d'Henri Mahé de Boislandelle : «Gestion des ressources humaines dans les P.M.E.»
}

\author{
Bruno $F A B I$, avec la collaboration \\ de Denis GARAND \\ Université du Québec à Trois-Rivières
}

\section{Analyse de l'ouvrage}

La Francophonie accueilleraavec intérêtl'ouvrage consacréà la Gestion des Ressources Humaines (G.R.H.) en contexte de P.M.E. (1988). Le professeur Mahé a en effet le mérite d'être le premier chercheur francophone à publier un volume complet visant à mieux comprendre et décrire la Fonction R.H. dans les P.M.E. Il désire ainsi pallier au manque d'intérêt et de publications manifesté jusqu'ici par les conseillers d'entreprises et les universitaires en sciences de la gestion à l'égard de cette fonction du management en P.M.E. Une telle publication semble donc répondre à un besoin chez les chercheurs comme chez les praticiens et elle s'inscrit dans un mouvement plus international, puisqu'elle suit la parution d'un ouvrage similaire en contexte américain (Arthur, 1987). suivants :

L'auteur poursuit un certain nombre d'objectifs qui peuvent se résumer aux

1. Partir d'une observation des pratiques en G.R.H. en moyennes (M.E.) et petites entreprises (P.E.) afin d'appuyer ses réflexions et ses propositions sur l'existant;

2. Resituer la G.R.H. dans le processus général de gestion de l'entreprise de façon à juger de son importance effective;

3. Sensibiliser les dirigeants et les cadres de M.E. et P.E. aux spécificités de la G.R.H. dans leurs unités de travail et les aider à mettre en place des processus de gestion adaptés; 
4. Alerter les étudiants en G.R.H. afin qu'ils ne focalisent pas uniquement leur attention sur les grandes entreprises qui disposent de moyens, de procédures et de méthodologies complexes, mais également sur les nombreuses M.E. s'interrogeant sur la pertinence et embauchant effectivement un responsable des R.H.;

5. Suggérerà des chercheurs en sciences de la gestion une piste riche en potentialités dans la mesure où les R.H. doivent être intégrées au technique et à l'économique afin de les rendre plus crédibles et surtout de les situer à leur véritable place dans l'entreprise, c'est-à-dire au centre.

Pour atteindre ces objectifs, le professeur Mahé nous propose un ouvrage constitué de 13 chapitres répartis dans 2 parties principales, elles-mêmes subdivisées en 5 sous-parties. De nombreuses subdivisions secondaires sont présentées dans une longue table des matières à la fin de cet ouvrage.

La première partie est consacrée à la justification de la nécessité d'intégrer la G.R.H. au système général de la gestion d'entreprise.

Une première sous-partie, constituée de 3 chapitres, examine l'état des pratiques en G.R.H. dans les P.M.E. à partir d'études de terrain réalisées en 1984 et 1985 dans des P.M.E. françaises. On s'y attarde notamment à l'organisation générale de la fonction personnel, aux modalités de prise en charge selon la taille des entreprises (T.P.E., P.E., M.E., M.G.E.) et aux améliorations empiriques de cette fonction du management en contexte de P.M.E. Parmi les nombreux résultats et propositions contenus dans cette première sous-partie, il semble opportun d'en relever quelques-uns :

1. La structuration plus ou moins formelle de la fonction personnel dans les P.M.E. dépend de plusieurs variables : taille, système de transformation, localisation, statut juridique et âge de l'entreprise, ainsi que personnalité du dirigeant, notamment sa formation initiale et sa hiérarchisation des fonctions de l'entreprise.

2. La fonction personnel a une position centrale dans l'entreprise, mais n'occupe pas dans l'esprit des dirigeants une place prépondérante.

3. Son importance réelle est sous-estimée car sa situation organisationnelle se confond souvent avec l'exercice d'autres fonctions administratives générales.

4. Son émergence formelle est très directement liée à la taille des entreprises et aux franchissements de seuils d'effectifs (au-delà de 200 salariés).

5. Derrière les apparences de sa faible structuration et de son statut de fonction secondaire, on doit remarquer que sa prise en charge réelle repose le plus souvent sur deux personnages centraux des P.M.E., à savoir le dirigeant et le comptable, (hormis les quelques cas où un responsable des R.H. est désigné).

6. Sur le plan des pratiques, le dirigeant délègue souvent les divers domaines d'administration du personnel, les aspects administratifs souvent prédominants 
en P.M.E., mais il se réserve toujours le recrutement et la rémunération qui revêtent donc à ses yeux un caractère essentiel.

7. Quelques indicateurs de dysfonctionnement montrent que les choses ne vont pas toujours très bien en matière de G.R.H. (absentéisme, rotation) et que les conditions de travail et de rémunération s'avèrent généralement moins bonnes que dans les grandes entreprises (G.E.).

La deuxième sous-partie, regroupant les chapitres 4 et 5 , est consacrée à la place des R.H. dans le processus général de gestion. Dans sa position d'analyste externe à l'organisation, l'auteur tente de faire avancer la réflexion en situant la G.R.H. dans les processus de formulation et de mise en place d'une stratégie dans les P.M.E. Pour étayer sa réflexion théorique, le professeur Mahé fait appel à des connaissances relevant de différents champs disciplinaires connexes à la G.R.H. : gestion stratégique, typologies des dirigeants, formules de gestion participative, théories des organisations et théories de la motivation. Cette longue démonstration amène l'auteur à formuler deux propositions qui lui semblent fondamentales :

1. La G.R.H. ne peut être considérée comme un rajout aux autres domaines de la gestion de l'entreprise, mais au contraire, comme une fonction capitale au coeur de tout le processus stratégique de l'entreprise.

2. Au niveau des P.E. et M.E., la stratégie de mise en place d'une véritable politique de G.R.H. devra d'abord passer par le dirigeant.

Cette réflexion théorique, associée aux observations des pratiques rapportées en première sous-partie, conduit l'auteur à proposer un modèle général de G.R.H.bâti autour d'un mix social. Ce modèle constitue indéniablement la pièce maîtresse de cette troisième sous-partie. Il s'impose en fait comme une des contributions majeures de cet ouvrage. Il semble par conséquent indiqué de le reproduire à la figure 1 (p. 102).

Ce modèle se veut clarificateur et simple. Il vise à intégrer et à regrouper l'arsenal de recettes, de méthodes et d'outils dans une succession de séquences cohérentes: actions, effets, évaluations, diagnosticset choix.S' inspirant del'approche systémique, l'auteur propose quatre grands axes politiques de G.R.H., susceptibles à la fois de couvrir l'ensemble des domaines et de former la base d'un processus global de régulation. Ces axes principaux et complémentaires sont les politiques d'emploi, de rémunération, de valorisation et de participation. Toutes les orientations et actions contenues dans les politiques de ce «mix de G.R.H.» devront être cohérentes, non seulement entre elles, mais aussi avec les autres choix technicoéconomiques de l'entreprise. L'évaluation de cette cohérence, et de l'efficacité du mix de G.R.H., nécessitera la prise en considération de différentes variables de résultats assorties d'un nombre plus ou moins grand d'indicateurs. A cet égard, le professeur Mahé propose sept variables de résultats, à savoir quatre effets directs (emploi, rémunération, valorisation et participation) et trois effets indirects (climat 


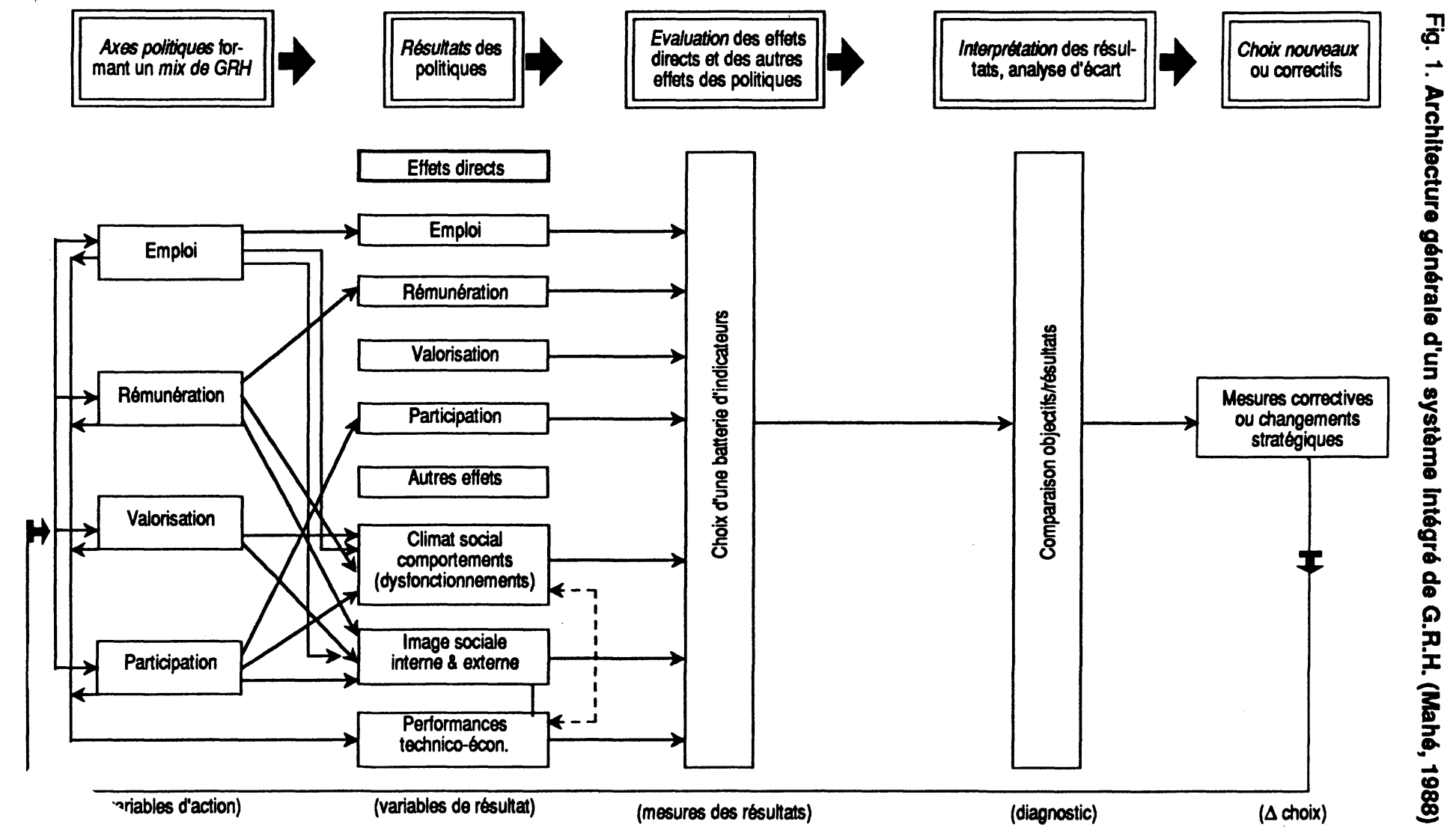


social, image sociale et performances technico-économiques). Il avance que son modèle peut, par sa conception et sa généralité, intégrer les spécificités et diversités des P.E. et M.E.

La deuxième partie de son ouvrage est consacré à cette démonstration. L'auteur y expose la mise en oeuvre d'une politique intégrative en G.R.H. Globalement, il traite des cinq phases d'opérations composantes de son modèle. Il développe tout d'abord l'ensemble des variables d'action en les regroupant autour de quatre grands axes politiques essentiels à la G.R.H. Ensuite, il présente les modalités d'évaluation des politiques et de leurs effets avec l'aide d'indicateurs qui permettront le diagnostic ou l'audit social et la suggestion de politiques correctrices.

Une première sous-partie porte donc sur les grands axes politiques de la G.R.H. Constituée de quatre chapitres, cette sous-partie aborde respectivement les politiques d'emploi, de rémunération, de valorisation et de participation. De nature plus descriptive, ces chapitres nous introduisent au coeur de la fonction R.H. en contexte de P.M.E. Le titre de l'ouvrage pouvait d'ailleurs nous laisser croire qu'il s'agissait là de l'essentiel de l'exposé. Pour cette raison, et pour faciliter la compréhension du lecteur, un résumé du contenu de ces quatre chapitres est présenté au schéma 1 (p. 104).

La consultation de ce schéma permet de constater une certaine constante au niveau du contenu de ces quatre chapitres. On y présente d'abord les éléments de connaissances traditionnelles (démarches, méthodes, techniques) relatives aux différentes pratiques de G.R.H. En l'occurence, la politique d'emploi regroupe les pratiques de planification des R.H., de recrutement et de sélection. La politique de rémunération reprend des notions d'évaluation d'emplois, de systèmes de salaire et d'intéressement ainsi que des avantages sociaux; on aborde également la question des coûts des R.H. La politique de valorisation reprend des notions d'évaluation du rendement (personnel), de formation et d'amélioration des conditions de travail. Finalement, la politique de participation emprunte des éléments de connaissances aux systèmes d'information, de communication et aux nouvelles formes de gestion participative, notamment les cercles de qualité. Certaines particularités des P.E. et M.E. sont soulevées. L'auteur présente également plusieurs aspects législatifs et règlementaires encadrant certaines pratiques de G.R.H. en contexte français. Il mentionne, en conclusion, que de nombreuses M.E. et P.E. limitent leurs efforts aux politiques d'emploiet de rémunération, négligeant parfois les éléments de valorisation et de participation.

Les deux derniers chapitres constituent la deuxième sous-partie de cette deuxième partie de l'ouvrage. Ils sont essentiellement consacrés à l'évaluation des résultats des quatre axes politiques de G.R.H., évaluation susceptible d'amener certaines mesures correctrices. On y retrouve notamment la description d'outils d'analyse traditionnels tels que les histogrammes, les diagrammes de Pareto, les tableaux à double entrée et les diagrammes de cause-effet. L'auteur présente également différents indicateurs permettant d'évaluer les effets directs et indirects de 


\begin{tabular}{|c|c|c|c|}
\hline $\begin{array}{l}\text { POLMRQUE } \\
\text { D'EMPLOI }\end{array}$ & $\begin{array}{c}\text { POLUMOUE } \\
\text { DE RENUNERATION }\end{array}$ & $\begin{array}{c}\text { POUMRQUE } \\
\text { DE VALORISATION }\end{array}$ & $\begin{array}{c}\text { POUTRQUE } \\
\text { DE PARTIPATION }\end{array}$ \\
\hline \multirow{2}{*}{$\begin{array}{l}\text { Notion d'effectif } \\
\text { analyse des effectifs } \\
\text { variation de l'emploi } \\
\text { évolution de la structure } \\
\text { stabilité du personnel }\end{array}$} & Contraintes & & Communication on PE of ME \\
\hline & \begin{tabular}{|l|} 
déterminants Kégaux de la fixation du salaire \\
mode de paiement du salaire \\
charges sociales obligatoires
\end{tabular} & \multirow{3}{*}{$\begin{array}{l}\text { évaluation du persomel } \\
-\quad \text { méthodes employées } \\
-\quad \text { évaluation des subordonnes } \\
-\quad \text { évaluation des cadres } \\
\text { politiques promotionnelles of de carriere }\end{array}$} & \multirow{8}{*}{ 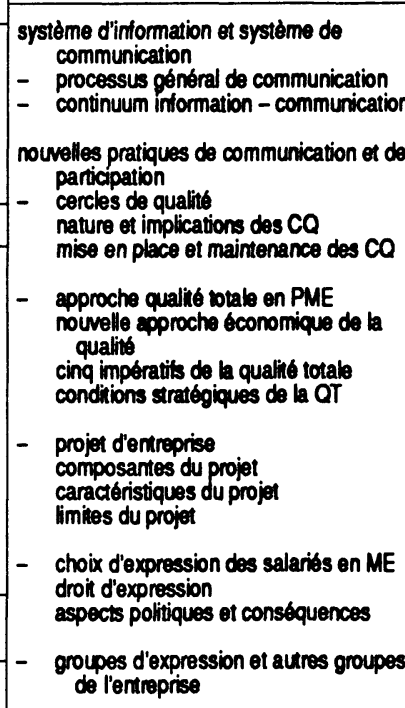 } \\
\hline Besoins of ressources en personnel & Système de rémunération & & \\
\hline \multirow{3}{*}{$\begin{array}{l}\text { Politiques d'ajustements intemes } \\
\text { et de maintien de l'emploi } \\
\text { duré du travail } \\
\text { modernisation d'equipement } \\
\text { rérientation stratégique limitee }\end{array}$} & \multirow{7}{*}{ 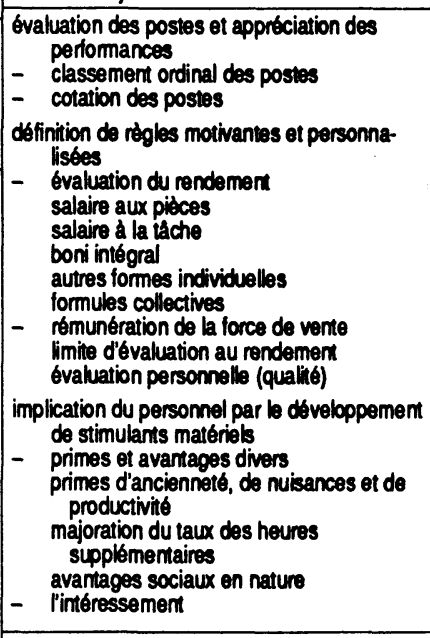 } & & \\
\hline & & Formation du personnel & \\
\hline & & \multirow[b]{2}{*}{ 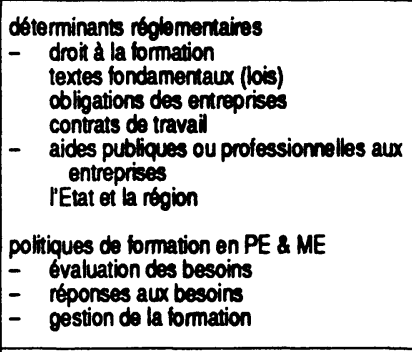 } & \\
\hline \multirow{6}{*}{ 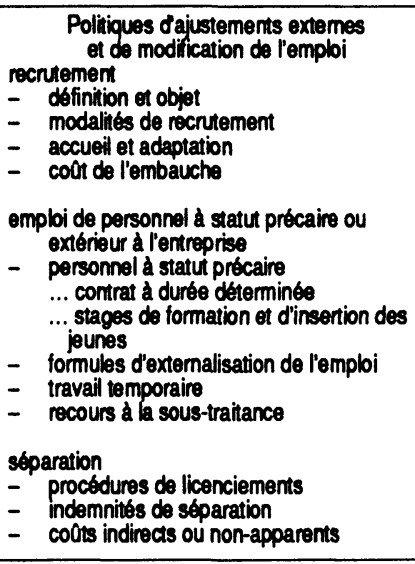 } & & & \\
\hline & & \begin{tabular}{|c|}
$\begin{array}{c}\text { Amétioration des conditions } \\
\text { de travail }\end{array}$ \\
\end{tabular} & \\
\hline & & \multirow{5}{*}{$\begin{array}{l}\text { aménagement du temps de travail } \\
-\quad \text { horaires classiques aménagés } \\
-\quad \text { horaires atypiques } \\
-\quad \text { incertitudes et perspectives } \\
\text { aménagement sur les conditions psychiques } \\
\text { du travail } \\
-\quad \text { ergonomie } \\
-\quad \text { aspects psycho-physiologiques } \\
\text { politiques d'hygiène of de sécurité } \\
-\quad \text { cadre réglementaire } \\
-\quad \text { pratique et colts }\end{array}$} & \\
\hline & & & $\begin{array}{l}\text { Participation } \\
\text { contre-pouve }\end{array}$ \\
\hline & Le cout des ressources humaines & & \multirow{3}{*}{ rolle des représentants du personnel } \\
\hline & \multirow{2}{*}{$\begin{array}{l}\text { rémunération et charges associées } \\
-\quad \text { rémunérations décidées } \\
- \text { charges imposées } \\
- \text { - masse salariale } \\
\text { coots d'ajustement du personnel } \\
\text { embauche, formation, animation, } \\
\text { séparation, remplacement et dysfonction- } \\
\text { nement } \\
\text { frais de gestion du personnel }\end{array}$} & & \\
\hline Attitude à l'égard de l'embauche & & & \\
\hline
\end{tabular}


ses axes politiques de G.R.H. Le lecteur averti en G.R.H. retrouvera donc des indicateurs habituels tels que les taux de roulement (rotation), d'évolution de masse salariale, d'absentéisme, de conflits, de productivité et de valeur ajoutée. De tels indicateurs supporteront un diagnostic ou un audit social, interne ou externe, permettant aux responsables de P.M.E. d'opéreréventuellement des choix d'orientation ou de correction à partir des quatre axes politiques de G.R.H.

Dans une conclusion générale plutôt succinte, le professeur Mahé énonce certaines perspectives de G.R.H. en P.M.E. Il termine par un plaidoyer en faveur de la poursuite des efforts de recherche et de sensibilisation des intervenants à la G.R.H. en P.M.E. Il nous met finalement en grande face à l'illusion de transposabilité des outils de la grande entreprise à la P.M.E. Quelques annexes et une bibliographie, majoritairement composée de titres français, complètent cet ouvrage.

\section{Une critique}

La description détaillée consacrée à ce volume témoigne de son importance pour une fonction du management encore très peu explorée en contexte de P.M.E. A cet égard, le professeur Mahé semble clairement avoir atteint plusieurs des objectifs qu'il s'était fixés. Il réussit effectivement à appuyer ses réflexions et ses propositions sur l'existant, sur des observations portant sur les pratiques de G.R.H. en contexte de P.E. et ME. Ses premiers chapitres contiennent des données empiriques dont on ne retrouve que très rarement l'équivalent dans la documentation internationale dans la mesure où elles reflètent le contexte réel des P.M.E. avec leurs contraintes matérielles et administratives. Cette bonne connaissance du terrain alimente avantageusement sa réflexion théorique qui débouche sur son modèle général de G.R.H. construit autour de quatre axes politiques principaux : emploi, rémunération, valorisation et participation. Il s'agit indéniablement là d'une contribution importante pour les recherches futures sur la fonction R.H. en P.M.E. Ce modèle regroupe et intègre non seulement plusieurs pratiques de G.R.H. autour de ces quatre axes principaux, mais il nous sensibilise à l'importance de resituer cette fonction dans le processus général de gestion. C'est ainsi que l'auteur propose des indicateurs permettant l'évaluation non seulement des effets directs des pratiques en G.R.H., mais également des effets indirects tels que le climat social, l'image sociale et les performances technicoéconomiques. Sa démonstration nous sensibilise et en fait nous convaint de l'importance primordiale que devrait avoir la fonction R.H. dans le processus stratégique en P.M.E.

Cependant, même un ouvrage de cette qualité n'échappe pas entièrement à la critique ou du moins à l'interrogation du lecteur. On se surprend d'abord de la structure globale du volume qui s'appréhende difficilement à cause d'une table des 
matières d'une lourdeur et d'une complexité qui se justifient mal dans un ouvrage de cette ampleur. Notre surprise s'avère encore plus grande devant un développement disproportionné des aspects stratégiques par rapport aux aspects techniques et opérationnels des pratiques en G.R.H. Malgré ce que le titre laisse entendre, on ne consacre que quatre des treize chapitres aux axes politiques en G.R.H., alors que deux longs chapitres sont consacrés à la place des R.H. dans la formulation et la mise en oeuvre de la stratégie en P.M.E. Une telle insistance amène l'auteur à juxtaposer des résumés et des énumérations relatives à la stratégie d'entreprise, à la théorie des organisations, aux theories de la motivation, à des typologies de dirigeants et à des formules participatives en P.M.E. Le lecteur reste avec une impression d'hétérogénéité où l'on dégage difficilement l'intégration et le fil conducteur. Cette insistance sur les aspects stratégiques se fait un peu au détriment des aspects opérationnels où l'on note certaines omissions fort discutables. A titre d'illustration, on relève, au chapitre de l'emploi, des carences évidentes en ce qui concerne l'entrevue de sélection et l'analyse des emplois. La même évaluation vaut pour les objectifs, les obstacles et les indicateurs en évaluation du rendement (personnel), au chapitre de la valorisation. En somme, on semble négliger certains aspects opérationnels qui nous semblent essentiels pour l'application efficace de certaines pratiques de G.R.H. aussi bien en contexte de P.M.E. que de G.E.

On regrette également la prédominance des aspects législatifs et règlementaires dans les quatre chapitres consacrés aux axes politiques de G.R.H. L'auteur semble justifier cette prédominance par «la législation, très contraignante en France, qui supplée largement dans l'esprit de beaucoup à la réflexion en la matière, jugée de ce fait inutile» (p. 228). La spécificité de ces lois et règlements ne peut que limiter l'universalité de l'ouvrage et risque d'amoindrir l'intérêt de plusieurs lecteurs francophones provenant de l'extérieur de la France. Ce déséquilibre se concrétise notamment au chapitre de la formation où l'on réserve le double de l'espace à des constatations d'ensemble d'ordre technique et légal, par rapport à des questions opérationnelles comme l'évaluation des besoins et la gestion de la formation. Ceci nous parait d'autant plus surprenant que l'auteur lui-même reconnaît que «se pose bien sûr plus fondamentalement pour chaque entreprise la question des besoins et des réponses à apporter de manière spécifique» (p. 217).

On ne saurait non plus passer sous silence certaines limites méthodologiques relevées dans les premiers chapitres faisant état d'études sur le terrain dans des P.M.E. françaises, dont certaines du Midi de la France. Nous aurions apprécié obtenir davantage d'informations relatives aux méthodes de cueillette et d'analyse des données, ainsiqu'àcertaines caractéristiques socio-démographiques des échantillons utilisés. De telles informations auraient possiblement permis de comprendre par exemple la non concordance entre les nombres d'entreprises rapportées dans les deux premiers chapitres.

L'auteur formule par ailleurs une critique fort discutable àl'égard des divers ouvrages consacrés à la G.R.H. où il relève une «liste impressionnante de rubriques, 
de chapitres, de sujets, de méthodes plus précis les uns que les autres» mais qui lui laissent «un sentiment de diversité, d'hétérogénéité, de succession de problèmes et de recettes formant un catalogue plus ou moins complet et plus ou moins pratique, ou théorique, selon les cas» (p. 139). Un tel commentaire nous semble ignorer l'apport de certains auteurs nord-américains qui ont déjà proposé des modélisations de la fonction R.H. inspirées de l'approche systémique (Bélanger, Petit et Bergeron, 1983) ou qui ont déjà procédé à des catégorisations et des regroupements des différentes pratiques de G.R.H. s'apparentant à certains égards aux quatre axes politiques de G.R.H. présentés dans cet ouvrage (Cascio, 1986; Milkovich et Glueck, 1985; Stone et Meltz, 1983). Il ne s'agit donc pas de minimiser l'apport du professeur Mahé dans son effort d'intégration de la fonction R.H., mais plutôt de nuancer son évaluation de l'état actuel des connaissances dans cette fonction du management.

Un dernier commentaire se veut davantage une interrogation fondamentale qu'une critique. En quoi consistent exactement les fameuses «particularités» de la fonction R.H. en contexte de P.M.E. ? L'auteur y fait quelquefois allusion dans son ouvrage et il conclut en formulant un plaidoyer en faveur de la poursuite des efforts de recherche visant à mieux cerner ces particularités. Ceci l'amène d'ailleurs à nous mettre en garde contre «l'illusion de transposabilité des outils de la grande entreprise» (p. 297). Pourtant, son volume ne contribue pas clairement à mettre cette illusion en évidence. Les résultats de recherche contenus dans les premiers chapitres font ressortir certaines «particularités» : des caractéristiques individuelles du dirigeant et des variables organisationnelles, dont la taille, semblent avoir un impact évident sur les niveaux de formalisation, de structuration et de prise en charge des pratiques en G.R.H. dans les P.M.E. Mais aucune donnée ne permet de supporter l'existence de différences ontologiques entre les pratiques de G.R.H. en P.M.E. et en G.E. En clair, les données révèlent la situation suivante : à mesure qu'une P.M.E. grossit, on observe une formalisation et une structuration des pratiques en G.R.H. qui seront éventuellement prises en charge par un responsable, et non plus uniquement par le dirigeant. Cette véritable G.R.H. sera intégrée à la stratégie de l'entreprise et s'éloignera des aspects administratifs et routiniers de l'administration du personnel. Pour y arriver, on devra, sauf preuve contraire, avoir recours aux outils et aux méthodologies de G.R.H. traditionnelles, celles-là mêmes que l'on utilise en G.E. A cetégard, il paraît significatif de constater que les quatre chapitres consacrés aux axes politiques de G.R.H. empruntent massivement et presqu'exclusivement aux connaissances normatives traditionnelles en G.R.H. L'illustration la plus frappante se retrouve probablement au chapitre de la participation où là la longue description des cercles de qualité constitue une duplication presqu'intégrale des éléments traditionnels relatifs au contenu et à la stratégie d'implantation de cette forme de gestion participative. La lecture de cet ouvrage laisse donc entières ces questions fondamentales ? La transposition des outils et des méthodologies de G.R.H., de la G.E.à la P.M.E., constitue-t-elle vraiment une illusion? Ne s'agit-il pas plutôt d'une piste intéressante à investiguer en concentrant nos énergies, non pas sur les méthodes ou les outils, mais plutôt sur les adaptations nécessaires aux contraintes financières 
et administratives des P.M.E. ? Il s'agit là d'hypothèses que l'état actuel de nos connaissances ne permet pas de rejeter à moins de sombrer dans un dogmatisme incompatible avec la démarche scientifique.

De telles interrogations confirment la pertinence de l'ouvrage du professeur Mahé. Il contribue en effet à stimuler l'intérêt pour la recherche et l'analyse des pratiques de G.R.H. en P.M.E. Ne serait-ce que pour cette raison, son volume mérite d'être consulté par tous les acteurs intéressés à mieux comprendre et à proposer des innovations dans cette fonction du management en P.M.E. Nous pensons ici principalement à des consultants, des étudiants et des chercheurs se préoccupant des P.M.E. Il convient donc de saluer le travail de pionnier du professeur Mahé qui ouvre la voie aux chercheurs francophones désireux d'aborder la G.R.H. en P.M.E. à partir d'un modèle intégré et rigoureux.

\section{BIBLIOGRAPHIE}

Arthur, D., (1987), Managing Human Resources in Small and Midsized Companies, Amacom, New York.

Belanger, L., Petit, A. et Bergeron, J.-L., (1983), Gestion des Ressources Humaines : une approche globale et intégrée, Gaétan Morin, Montréal.

Cascio, W., (1986), Managing human resources, McGraw-Hill, New York.

Mahé de Boislandelle, H., (1988), Gestion des ressources humaines dans les P.M.E., Paris, Economica, 1988, 322 p.

Milkovich, G.T. et Glueck, W.F., (1985), PersonnelHHuman Resource Management : A Diagnostic Approach, Business Publications, Plano, Texas.

Stone, T.H. et Meltz, N.M., (1983), Personnel management in Canada, Holt, Rinehart and Winston, Toronto. 